\title{
Tuning the electronic and magnetic properties of graphene
}

\section{flake embedded in boron nitride nanoribbons with transverse electric fields: First-principles calculations}

\author{
Zhaoyong Guan, ${ }^{\dagger},{ }^{\ddagger}, *$ Shuang $\mathrm{Ni},{ }^{\xi}$ Shuanglin $\mathrm{Hu}^{\#}$ \\ $†$ School of Chemistry and Chemical Engineering, Shandong University, 250100 \\ Jinan, P. R. China \\ \$Department of Physics, Tsinghua University, Beijing 100084, P. R. China \\ ${ }^{\xi}$ Research Center of Laser Fusion, China Academy of Engineering Physics.
} Mianyang, Sichuan 621900, P. R. China

\# Institute of Nuclear Physics and Chemistry, China Academy of Engineering Physics, Mianyang, Sichuan 621900, P. R. China

1. The magnetic moment of 6-T1-C $3 \mathrm{~B} 1 \mathrm{~N}-6$ zigzag and $8-\mathrm{T} 2-\mathrm{C}_{3 \mathrm{~B} 6 \mathrm{~N}-8}$ armchair CBNNR. The magnetic moment mainly locates at the carbon atoms. And the nearby nitrogen atoms connected with carbon atoms also make little contribution.
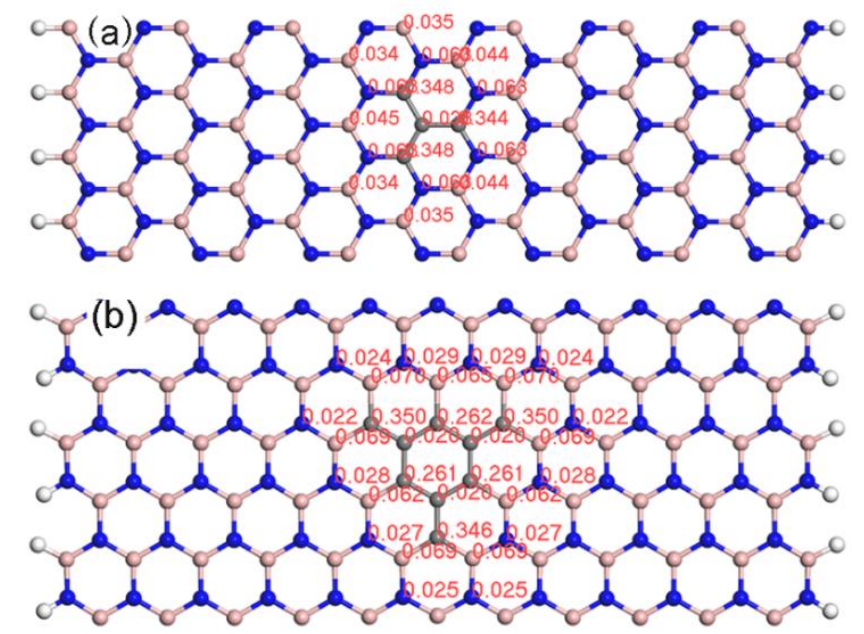
Figure S1. The magnetic moment distribution of (a) 6-T1-C3B1N-6 zigzag, (b) 8-T2$\mathrm{C}_{3 \mathrm{~B} 6 \mathrm{~N}-8}$ armchair CBNNRs, respectively. The figures show the magnetic moment of the corresponding atoms.

2. The band structure of 6-T1- $\mathrm{C}_{6 \mathrm{~B} 3 \mathrm{~N}}-5 \mathrm{z} \mathrm{CBNNR}$ under the E-field $=-0.081,-0.082$, $0.083,-0.084,-0.086,-0.089 \mathrm{~V} / \AA$. For $-0.081 \mathrm{~V} / \AA$, the system is SGS. For E-field $=-0.082--0.089 \mathrm{~V} / \AA$, they are all spin-polarized metal with Dirac cones at the fermilevel.

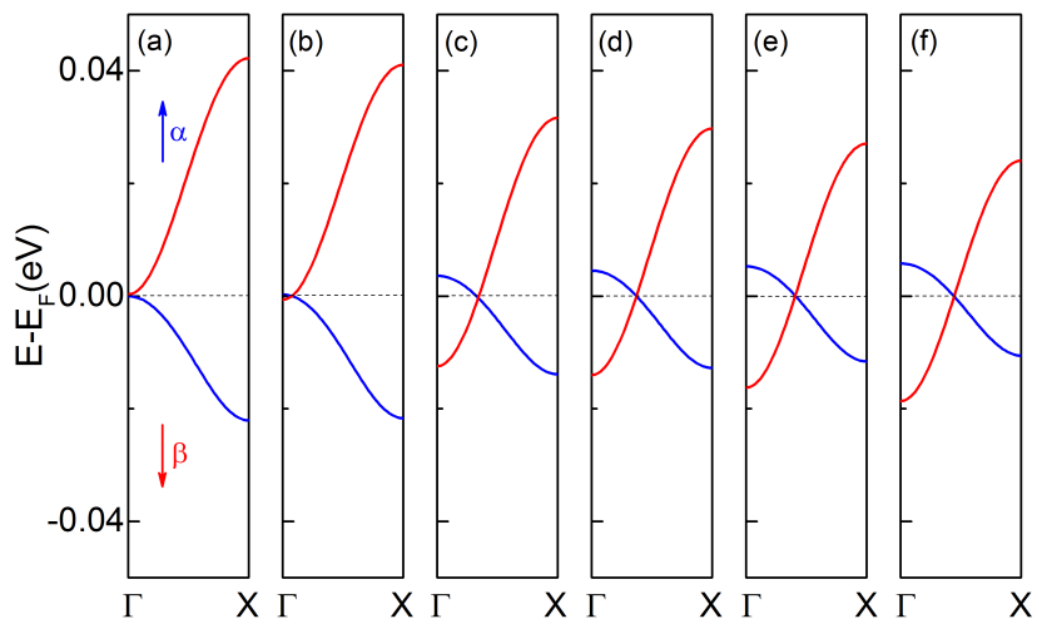

Figure S2. The band structure of 6-T1- $\mathrm{C}_{6 \mathrm{~B} 3 \mathrm{~N}}-5 \mathrm{ZCBNNR}$ under the E-field of (a) 0.081, (b) -0.082 , (c) -0.083 , (d) -0.084 , (e) -0.086 and (f) $-0.089 \mathrm{~V} / \AA$, respectively.

3. About the size and position of the graphene flakes affect the electronic properties of the CBNNRs.

First, the size of the graphene flake is related with the number of boron and nitrogen atoms replaced by carbon atoms. As the size of graphene flakes increases, the number of doped carbon atoms also increases. So the band gap of the boron nitride nanoribbons monotonously decreases. Second, the position of the graphene flake can modify the band gaps of boron nitride nanoribbons. Because the original boron nitride nanoribbons, the charge density of the VBM and CBM localize at the edge of the nanoribbons. When the graphene flake replace the edge boron and nitrogen atoms, the corresponding band gap $(0.599 \mathrm{eV})$ obviously decreases for introducing 
the doped states near the fermi-level. When the doped position is shifted away from the edge to the center, the corresponding band gap $(0.626 \mathrm{eV})$ is not as small as the edge ones. When the doped graphene flakes localize at the center of the BNNRs, the system has the bigger band gap $(0.708 \mathrm{eV})$. And it introduces the doped states to the whole system. Compared with the graphene flakes doped in the edge, the band gap becomes bigger $(0.708 \mathrm{eV})$.
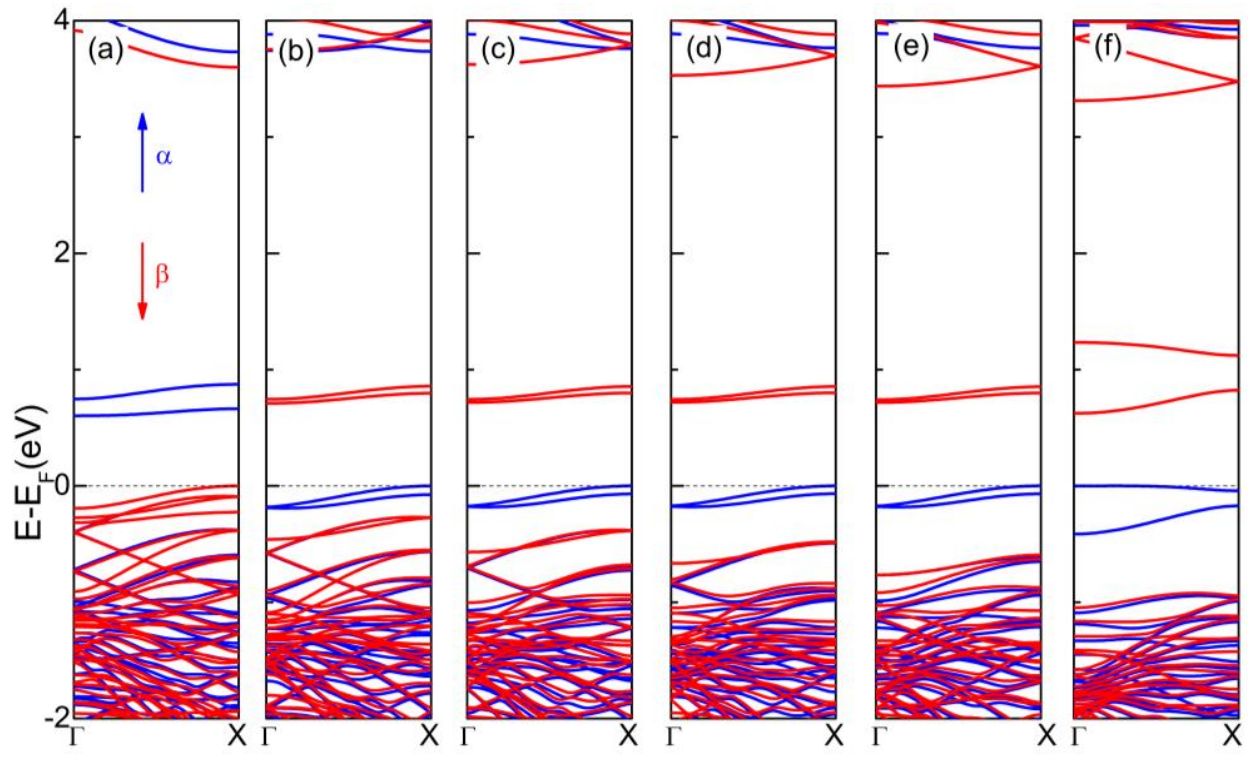

Figure S3. The band structure of the (a) $0-\mathrm{T} 1-\mathrm{C}_{1 \mathrm{~B} 3 \mathrm{~N}}-12$, (b) $1-\mathrm{T} 1-\mathrm{C}_{1 \mathrm{~B} 3 \mathrm{~N}}-11$, (c) $2-$

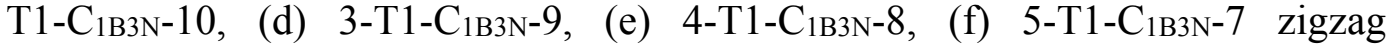
CBNNRs, respectively. 